\title{
On the importance of inner-shell transitions for opacity calculations
}

\author{
N R Badnell ${ }^{1}$ and M J Seaton ${ }^{2}$ \\ ${ }^{1}$ Department of Physics, University of Strathclyde, Glasgow G4 0NG, UK \\ ${ }^{2}$ Department of Physics and Astronomy, University College London, London \\ WC1E 6BT, UK
}

\begin{abstract}
For high temperatures and densities, stellar opacities obtained from the Opacity Project (OP) were smaller than those obtained from the OPAL project. Iglesias and Rogers [Astrophys. J. 443469 (1995)] suggested that the discrepancy was due to the omission by OP of important atomic inner-shell processes, and considered in detail results for a mixture of 6 elements: H, He, $\mathrm{C}, \mathrm{O}, \mathrm{S}$ and $\mathrm{Fe}$. Extensive new inner-shell data have now been computed using the code AUtOSTRUCTURE. It is shown that the inclusion of these data in the OP work gives opacities for the 6-element mix which are in much closer agreement with those from OPAL. We also discuss a number of problems relating to the calculation of opacities and of equations-of-state in dense plasmas.
\end{abstract}

Submitted to: J. Phys. B: At. Mol. Opt. Phys.

PACS numbers: 32.80.Hd, 95.30.Ky

\section{Introduction}

Energy is produced by nuclear reactions at the centre of a star, at a temperature of a few times $10^{7} \mathrm{~K}$, and escapes at the stellar surface. The structure of a star is determined by the equations for conservation of mass and of energy, an equation for hydrostatic equilibrium, and by the temperature gradient (see $[1,2,3])$. In regions for which convection does not occur, the temperature gradient is determined by the Rosseland-mean opacity, which is the concern of the present paper.

In a stellar interior, an atom of a chemical element $k$ can exist in a number of ionization stages, $i$, and energy levels, $j$. Using the frequency variable $u=h \nu /\left(k_{\mathrm{B}} T\right)$ (where $k_{\mathrm{B}}$ is the Boltzmann constant), let $\sigma_{i j k}(u)$ be the cross section for absorption $\S$ or scattering of radiation by level $(i j k)$ and let $p_{i j k}$ be the probability of that level being populated. Then, the opacity cross-section for element $k$ is

$$
\sigma_{k}(u)=\sum_{i, j} p_{i j k} \sigma_{i j k}(u) \text {. }
$$

With $f_{k}$ the fractional abundance for element $k$, normalized to

$$
\sum_{k} f_{k}=1
$$

$\S$ For absorption processes, the correction factor for stimulated emission, $[1-\exp (-u)]$, is included. 
the mean cross-section per atom for a mixture of chemical elements is

$$
\sigma(u)=\sum_{k} f_{k} \sigma_{k}(u) .
$$

The Rosseland-mean cross-section is $\sigma_{\mathrm{R}}$, where

$$
\frac{1}{\sigma_{\mathrm{R}}}=\int_{0}^{\infty} \frac{1}{\sigma(u)} F(u) \mathrm{d} u
$$

and

$$
F(u)=\left[15 /\left(4 \pi^{4}\right)\right] u^{4} \exp (-u) /[1-\exp (-u)]^{2}
$$

(see $[1,2,3])$. Astronomers usually use opacities per unit mass. The Rosseland-mean opacity per unit mass is

$$
\kappa_{\mathrm{R}}=\sigma_{\mathrm{R}} / \mu,
$$

where $\mu$ is the mean atomic weight.

The 1982 paper of Simon [4] suggested that possible errors in opacities might explain discrepancies between theory and observations for pulsational properties of stars, and provided the stimulus for two major new efforts in opacity calculations: one referred to as OPAL at the Lawrence Livermore National Laboratory by C. A. Iglesias and and F. J. Rogers; and the other referred to as The Opacity Project, OP. Early OPAL results [5] showed that the inclusion of very large numbers of spectrum lines did indeed lead to major revisions in opacities. A number of subsequent OPAL papers have been published, of which one of the most recent is [6]. The present paper is a continuation of the OP work. The calculations of atomic data were described in a series of papers in this journal, of which the first was [7], and OP results for opacities were published in [8], to be referred to as SYMP. A collection of papers from the OP work, together with selected atomic data tables, have been published in book form $[9]$.

For regions of stellar interiors with temperatures of a few times $10^{5} \mathrm{~K}$, which are of particular importance for pulsation studies, opacities from OPAL and OP are in close agreement and can be larger than values previously adopted, by up to factors of about 3. The new opacities have led to substantial improvements in the agreement between calculated and observed pulsation properties. However, for deeper layers, the results from OPAL were larger than those from OP, by amounts of $30 \%$ or so.

Very large numbers of spectrum lines are included in the calculations, of order a few times $10^{7}$ for a complete opacity run. OPAL used a parametric potential model [10], while OP (see [9]) used $R$-matrix calculations supplemented by data from Kurucz [11] and from superstructure [12]. Much of the OP data includes allowance for configuration-interaction effects which are not included in the OPAL work. In their 1995 paper [13], to be referred to as IR95, Iglesias and Rogers suggested that the discrepancies between OPAL and OP at the higher temperatures and densities occurring in the deeper layers of stellar interiors could be due to the omission by OP of some important inner-shell transitions. We have now made new calculations, using the code AUTOSTRUCTURE [14], for promotions of inner-shell electrons, both via photoionization and photoexcitation of autoionizing states. Our results confirm the essential correctness of the suggestion made in IR95. The present paper describes the new atomic-physics calculations and presents results for the 6-element mix of IR95.

The structure of the paper is as follows: in section 2 we discuss issues relating to the equation-of-state; in section 3 we detail the new inner-shell transitions that 
we now include and the methodology that we used to describe them; in section 4 we briefly discuss free-free transitions: in section 5 we present and discuss our results for Rosseland-mean opacities; in section 6 we discuss a number of issues that arise in determining opacities; in section 7 we look at the opacity in the solar centre region; and finally, in section 8 , we give a brief summary.

\section{The equation-of-state (EOS)}

The fraction of element $k$ in ionization stage $i$ is

$$
\phi_{i k}=\sum_{j} p_{i j k} .
$$

We may put

$$
p_{i j k}=\phi_{i k} \times g_{i j k} W_{i j k} \exp \left[-E_{i j k} /\left(k_{\mathrm{B}} T\right)\right] / U_{i k}
$$

where, for level $(i j k), g_{i j k}$ is statistical weight, $E_{i j k}$ the total energy and $W_{i j k}$ an occupation probability. The internal partition function is

$$
U_{i k}=\sum_{j} g_{i j k} W_{i j k} \exp \left[-E_{i j k} /\left(k_{\mathrm{B}} T\right)\right] .
$$

If all $W_{i j k}$ were set to unity, the summation for $U_{i k}$ would be divergent. Due to interactions with particles and fields of the surrounding plasma, states of sufficiently high energy have only small probabilities of being occupied and, hence, small values of $W_{i j k}$.

\subsection{Occupation probabilities}

The methods used in the OP work for the calculation of the $W_{i j k}$ are described by Hummer and Mihalas in [15]. Let $F$ be the ion micro-field and $P(F)$ the micro-field distribution (MFD): $P(F) \mathrm{d} F$ is the probability of $F$ being in the range $\mathrm{d} F$, with

$\int_{0}^{\infty} P(F) \mathrm{d} F=1$. Hummer and Mihalas define critical fields, $F_{i j k}$, such that species $(i j k)$ can only exist in an environment with $F<F_{i j k}$, giving

$$
W_{i j k}=\int_{0}^{F_{i j k}} P(F) \mathrm{d} F .
$$

The use of equation (10) gives $W \simeq 1$ for low densities but $W$ to be small for high states and high densities. In [15] Hummer and Mihalas used the Holtsmark MFD, which does not make full allowance for correlations between particles nor for the charge on the ion $(i j k)$. They also introduced a simple analytical approximation (equation (4.70) of [15]) which gave results in close agreement with those from (10) at low densities but a more rapid exponential decrease at high densities. A similar exponential form is obtained using a 'hard-sphere' approximation (see section III (a) of [15]). The analytical form, (4.70) of [15], was used in SYMP.

Iglesias and Rogers, in IR95, noted that the Holtsmark distribution would not be a good approximation at the higher densities of stellar interiors and Nayfonov et al. [16] subsequently obtained improved expressions for the $W_{i j k}$ using the micro-field distributions of Hooper [17], which are also used in the OP calculations of line-profiles for hydrogenic ions [18]. Following Nayfonov et al., we refer to the equation of state using their MFD as the Q-EOS. Their $W_{i j k}$ are fairly close to those obtained using the APEX distribution of IR95 and, with one modification to be described below, will be used in the present work. 


\subsection{The ionization equilibrium}

For simplicity of presentation, we here omit specification of the element index $k$ and neglect all refinements such as allowance for electron degeneracy. The conclusions reached remain valid when all necessary refinements are included. We take the ionization index $i$ to be equal to the number of bound electrons: $i=0$ for the bare nucleus and $i=Z$ for a neutral atom with nuclear charge $Z$.

The ratio of ionization fractions in successive stages is (see [7] or [15])

$$
\frac{\phi_{i}}{\phi_{i-1}}=\frac{U_{i}}{U_{i-1}} \times \frac{N_{\mathrm{e}}}{U_{\mathrm{e}}},
$$

where $N_{\mathrm{e}}$ is the electron density,

$$
U_{\mathrm{e}}=2\left\{\frac{m_{\mathrm{e}} k_{\mathrm{B}} T}{2 \pi \hbar^{2}}\right\}^{3 / 2}
$$

and $m_{\mathrm{e}}$ is the mass of an electron.

Since $U_{0}=1$, the fraction in stage $i$ relative to the fraction of bare nuclei is

$$
\frac{\phi_{i}}{\phi_{0}}=U_{i} \times\left[\frac{N_{\mathrm{e}}}{U_{\mathrm{e}}}\right]^{i} .
$$

\subsection{Pressure ionization}

Now consider fixed $T$ and increasing $N_{\mathrm{e}}$. Initially, $\phi_{i} / \phi_{0}$ will increase with increasing $N_{\mathrm{e}}$ due to the factor $\left[N_{\mathrm{e}} / U_{\mathrm{e}}\right]^{i}$ in (13). That effect is pressure recombination. Eventually, for large densities, the $W_{i j}$ in $U_{i}$ become small and, if they become sufficiently small, pressure recombination can be followed by pressure ionization.

For $W$ calculated using (10), in the limit of $N_{\mathrm{e}}$ large one obtains $W \propto N_{\mathrm{e}}^{-2}$ using the Holtsmark MFD and $W \propto N_{\mathrm{e}}^{-3 / 2}$ with the $\mathrm{Q}$ form. In either case it is seen that pressure ionization does not occur for $i \geq 2$. That result would appear to be quite unphysical, since it would imply that all atoms are in states which have very small occupation probabilities. Pressure ionization does occur, for all $i$, if one uses a 'hardsphere' model for $W$ or, as in SYMP, the approximation of equation (4.70) of [15], giving exponential decreases of $W$ with increasing $N_{\mathrm{e}}$.

We conclude that equation (10) can be expected to give reasonable values for $W$ when $W$ is not very small, but that it does not give a sufficiently rapid decrease of $W$ in the limit of high densities. We adopt the expedient of introducing a critical value $W_{\mathrm{c}}$ of $W$; use the value of $W$ from equation (10) if it is greater than $W_{\mathrm{c}}$; and take $W=0$ if equation (10) gives $W<W_{\mathrm{c}}$. For quite a wide range of values of $W_{\mathrm{c}}$, results for opacities are found to be insensitive to the value of $W_{\mathrm{c}}$ adopted. The final results reported in the present paper are obtained with $W_{\mathrm{c}}=10^{-3}$.

The need for introducing a cut-off in $W$ is illustrated in Figure 1 for carbon at rather high temperature $(\log (T)=7.5)$ and very high densities $\left(\log \left(N_{\mathrm{e}}\right)=27.0\right.$, 27.5 and 28.0). Figure 1 (a) shows ground-state occupation probabilities $W$ against ionization stage $i$. Neutral carbon, $i=6$, is seen to have very small values of $W$ which decrease with increasing $N_{\mathrm{e}}$. Figure 1 (b) shows ionization fractions calculated without a cut-off in $W$. There is seen to be an abrupt change from the case of $\log \left(N_{\mathrm{e}}\right)=27.0$ with carbon nearly fully-ionized (ionization fraction 0.98 for $i=0$ ) to the case of $\log \left(N_{\mathrm{e}}\right)=28.0$ with carbon nearly fully-neutral (ionization fraction 0.95 for $i=6$ ). The result for $\log \left(N_{\mathrm{e}}\right)=28$ is clearly nonsensical, with nearly all of the carbon in a 
state with occupation probability of $W \simeq 10^{-12}$ ! With a cut-off of $W_{\mathrm{c}}=10^{-3}$ we obtain a result which is much more plausible: for $\log \left(N_{\mathrm{e}}\right)=27.0$, ionization fractions very similar to those for $W_{\mathrm{c}}=0$ for $i \leq 3$ and equal to zero for $i>3$; and for $\log \left(N_{\mathrm{e}}\right)=27.5$ and 28.0 the carbon is fully ionized.

\subsection{The $W_{n}$ of $\mathrm{OPAL}$}

Bethe and Salpeter [19] give expressions for $\left\langle r_{n l}^{3}\right\rangle$ for hydrogenic ions of charge $Z$. We put

$$
\left\langle r_{n}^{3}\right\rangle=\left(\frac{1}{n^{2}}\right) \sum_{l=0}^{n-1}(2 l+1)\left\langle r_{n l}^{3}\right\rangle
$$

to obtain

$$
\left\langle r_{n}^{3}\right\rangle=\left[n^{2} /\left(8 Z^{3}\right)\right]\left[21 n^{4}+35 n^{2}+4\right] .
$$

The mean volume for state $n$ can be defined as

$$
V_{n}=(4 \pi / 3)\left\langle r_{n}^{3}\right\rangle .
$$

Let $N_{\mathrm{e}}$ be the electron density, $N_{a}$ be the atom density and $\rho$ be the mass density. From Table 1 and Figure 1 of IR95, $N_{\mathrm{e}}=5.0 \times 10^{21} \mathrm{~cm}^{-3}$ and, for $\log (\rho)=-2$ and the 6-element mix (see section 5.2), $N_{a}=4.7 \times 10^{21} \mathrm{~cm}^{-3}$. The total particle density is then $N=N_{\mathrm{e}}+N_{a}=9.7 \times 10^{21} \mathrm{~cm}^{-3}$. The average volume occupied by a particle (electron or atom) is $1 / N$.

Table 1 gives, for hydrogenic carbon and the case of Table 1 of IR95 $(\log (T)=6$, $\log (\rho)=-2)$ : values of $N V_{n} ; W_{n}(\mathrm{OPAL})$ from IR95; and $W_{n}(\mathrm{Q})$ from formulae given in [16]. The values of $W_{n}(\mathrm{Q})$ are fairly close to the values of $W_{n}(\mathrm{APEX})$, as given in IR95.

In both OP and OPAL, optical properties (oscillator strengths and photoionization cross sections) are calculated for unperturbed atomic states, and $W_{n}$ is the probability of state $n$ being unperturbed. With $N V_{n}>1$, one would expect to find at least one other plasma particle within the volume $V_{n}$, giving a state which is markedly perturbed and which one would therefore expect to have a small value of $W_{n}$. By that criterion, some values of $W_{n}(\mathrm{OPAL})$ seem to be surprisingly large, particularly those for $n=4,5$ and 6 . Values of $W_{n}(\mathrm{Q})$ appear to be more reasonable.

\section{Inner-shell atomic physics}

The original OP work utilized the $R$-matrix method which uses wavefunction expansions of the type

$$
\Psi=\mathcal{A} \sum_{n} \psi_{n} \theta_{n}
$$

where the $\psi_{n}$ are functions for atomic 'target' states, the $\theta_{n}$ are functions for an added electron, and $\mathcal{A}$ is an anti-symmetrization operator. In that method, photoionization and autoionization are treated as a single quantum-mechanical process. Figure 2 gives, as an example, the cross section for transitions from the $1 \mathrm{~s} 2 \mathrm{~s}{ }^{1} \mathrm{~S}$ state of $\mathrm{Fe}^{24+}$. Below the threshold for ejection of the 1s electron, the cross section shows autoionization features due to processes

$$
1 \mathrm{~s} 2 \mathrm{~s}+h \nu \rightarrow 2 \mathrm{~s} n \mathrm{p} \rightarrow 1 \mathrm{~s}+\mathrm{e}^{-} .
$$


However, use of the $R$-matrix method would not be practicable for the computation of the atomic data required for the present work, for two reasons: (a) in many cases, the number of channels $n$ required in equation (17) would be prohibitive (our experience with the RmaX work on inner-shell X-ray processes [20] shows that a few $\mathrm{Li}$ - and Be-like ions are the most that could be treated with a reasonable timescale); (b) it would be difficult to allow for pressure-broadening of the autoionization features. We therefore use a perturbative approach, as implemented in the program AUTOSTRUCTURE [14, 24].

We note that Figure 2 shows, qualitatively, the effects of interference between autoionization features and the background continuum. However, detailed quantitative studies [21] show that, on averaging over resonance profiles, this interference is a very small effect and it can safely be neglected for our purposes. This is the independent processes approximation. In the time-reversed case, this corresponds to treating dielectronic and radiative recombination separately. The second approximation required by our perturbative approach is an isolated resonance treatment of the autoionizing features. The effect of interacting resonances has been investigated [21] for the reverse process of dielectronic recombination, and it also can safely be neglected for our purposes.

\subsection{Photoexcitation}

The downward probability rate for a radiative transition from an upper state $u$ to a lower state $l$ is given by

$$
A_{u \rightarrow l}^{\mathrm{r}}=\frac{1}{g_{u}} \frac{4 \omega^{3}}{3 \hbar c^{3}} S_{l u},
$$

where $g_{u}$ is the statistical weight for the upper level, $c$ is the speed of light, $\omega=2 \pi \nu$ where $\nu$ is the photon frequency, and $S_{l u}$ is the bound-bound line-strength, as defined in [22]. Eissner et al [23] give expressions for $S_{l u}$ in multi-configuration $L S$ - and intermediate-coupling (their equations (115) and (117), respectively): their code, SUPERSTRUCTURE, gives numerical values for $S_{l u}$ in atomic units. The code AUTOSTRUCTURE, which incorporates SUPERSTRUCTURE, gives the following data: (a) the line-centre frequency; (b) the rates $\left(g_{u} / g_{l}\right) A_{u \rightarrow l}^{\mathrm{r}} ;$ (c) $A_{u}^{\mathrm{r}} \equiv \sum_{l} A_{u \rightarrow l}^{\mathrm{r}}$, the total probability rate for radiative decay of the upper level $u$; (d) $A_{u \rightarrow m}^{\mathrm{a}}$ and $A_{u}^{\mathrm{a}} \equiv$ $\sum_{m} A_{u \rightarrow m}^{\mathrm{a}}$, the partial and total autoionization decay probability rates, respectively. Multi-configuration $L S$ - and intermediate-coupling expressions for $A_{u \rightarrow m}^{\mathrm{a}}$ are given in [14] (equations (2.2) and (2.4), respectively). The rates are all in $\mathrm{s}^{-1}$.

The oscillator-strength for the $l \rightarrow u$ transition is

$$
f_{u l}=\frac{m_{\mathrm{e}} c^{3}}{2 e^{2} \omega^{2}} \frac{g_{u}}{g_{l}} A_{u \rightarrow l}^{\mathrm{r}}
$$

and the absorption (photoexcitation) cross section for an autoionizing feature is

$$
\sigma_{l \rightarrow u}^{\mathrm{PE}}=\frac{2 \pi^{2} e^{2}}{m_{\mathrm{e}} c} f_{u l} \varphi(\omega),
$$

where $\varphi(\omega)$ is the line-profile factor. On neglecting thermal Doppler broadening, the profile is

$$
\varphi(\omega)=(\Gamma / 2 \pi) /\left[\left(\omega-\omega_{0}\right)^{2}+(\Gamma / 2)^{2}\right],
$$

where $\omega_{0}$ is the line-centre angular frequency and

$$
\Gamma=\left(A_{l}^{\mathrm{r}}+A_{u}^{\mathrm{r}}\right)+A_{u}^{\mathrm{a}}+\Gamma_{l u}^{\mathrm{p}},
$$


where expressions for the pressure-broadening contribution, $\Gamma_{l u}^{\mathrm{p}}$, are given in [35] (a different form is used for hydrogenic lines, see [18]). The profile (22) is then convolved with that for Doppler broadening to give a Voigt profile.

For more detailed (collisional-radiative) modelling purposes we require to follow the break-up of an autoionizing state. For example, the photoexcitation cross section in equation (21) is multiplied by branching ratios for radiative decay, $A_{u \rightarrow l}^{\mathrm{r}} /\left(A_{u}^{\mathrm{r}}+A_{u}^{\mathrm{a}}\right)$, and by Auger yields for autoionizing decay, $A_{u \rightarrow m}^{\mathrm{a}} /\left(A_{u}^{\mathrm{r}}+A_{u}^{\mathrm{a}}\right)$.

\subsection{Photoionization}

The direct photoionization cross-section from an initial state $l$ of an $(N+1)$-electron atom to a final state $u$ of an $N$-electron ion plus ejected electron is given by [7]

$$
\sigma_{l \rightarrow u}^{\mathrm{PI}}=\frac{1}{g_{l}} \frac{4 \pi^{2}}{3 c} \omega S_{u l},
$$

where $g_{l}$ is the statistical weight of the initial level and $S_{u l}$ is the bound-free linestrength with the final-state continuum wavefunction normalized per unit energy. The code AUTOSTRUCTURE uses a different continuum normalization and gives numerical values for the bound-free line strength which are equal to $\pi / 2$ times $S_{u l}$ in atomic units. The same expressions for $S_{u l}$ that were used for bound-bound transitions are used for bound-free transitions, except that the final 'active' bound-state wavefunction is replaced by a continuum distorted-wave function which does not contain any resonance structure. This use of distorted-waves is a good approximation for atoms that are a few times ionized and is the final approximation employed by our perturbative approach.

\subsection{AUtostructure, some details}

3.3.1. Angular algebra AUTOSTRUCTURe $[14,24]$ incorporates SUPERSTRUCTURE [23] and the angular algebra required to calculate the preceding atomic data is no more than that which is generated by SUPERSTRUCTURE to determine energy levels and radiative rates. (The angular algebra required for autoionization rates is that which follows from the $H$ operator which determines the structure.) However, for the complex inner-shell processes considered here, which can give rise to configurations with thousands of terms, we found it necessary to re-write the angular algebra code. Specifically, because of historical memory limitations, the algebraic Slaterstate interactions were determined between each $L S$ term or $J$ level [23]. However, the Slater-state interaction depends only trivially on the configuration and can be generated much more efficiently between symmetry groups. With complex configurations there is a high degree of algebraic term and level degeneracy, both within and between configurations. Re-coupling by $L S$ or $L S J$ symmetry groups reduces the overall time spent on the largest scale jobs by a factor of 30-40 $(L S)$ or 100-200 (LSJ).

3.3.2. The Hamiltonian matrix SUPERSTRUCTURE determines multi-configuration eigenenergies and eigenvectors by diagonalization of the Hamiltonian matrix for each $S L \pi$ or $J \pi$ symmetry group. AUTOSTRUCTURE further partitions the problem by $(N+1)$-electron bound and $N$-electron (plus continuum) configurations - the $N$ and $(N+1)$-electron Hamiltonians are diagonalized separately. The bound-free Hamiltonian interaction between the two is treated as a perturbation - this leads simply to the autoionization rate [14]. 
The mass-velocity and Darwin operators of the Breit-Pauli Hamiltonian [23] are also added to the usual non-relativistic Hamiltonian for the determination of our $L S$ coupling atomic structure. This results-in transition energies between terms which are in good agreement with those obtained from using intermediate coupling, on averagingover fine-structure.

\subsection{Application to the 6-element mix}

The $K$-shell processes required are of the form:

$$
1 \mathrm{~s}^{q} 2 l^{p} n^{\prime} l^{\prime}+h \nu \rightarrow 1 s^{q-1} 2 l^{p} n^{\prime} l^{\prime}+\mathrm{e}^{-}
$$

for photoionization and

$$
1 \mathrm{~s}^{q} 2 l^{p} n^{\prime} l^{\prime}+h \nu \rightarrow 1 \mathrm{~s}^{q-1} 2 l^{p} n^{\prime} l^{\prime} n^{\prime \prime} l^{\prime \prime} \rightarrow 1 s^{q-1} 2 l^{p} n^{\prime \prime \prime} l^{\prime \prime \prime}+\mathrm{e}^{-}
$$

for photoexcitation-autoionization, where $2 l^{p}$ stands for $2 \mathrm{~s}^{s} 2 \mathrm{p}^{t}$ with $p=s+t$. Calculations are made for $q=1,2$ for $p=0$ and $q=2$ for $p>0$. The values of $p$ depend on the element, for example, up to $p=7$ for iron but only $p=1$ for carbon. This depends on the importance of its contribution to the opacity. We use $n^{\prime}, n^{\prime \prime}, n^{\prime \prime \prime}=2$ to 6 , for all allowed $l^{\prime}, l^{\prime \prime}, l^{\prime \prime \prime}$. The contributions from higher- $n$ in (26) are obtained by matching onto the results of (25).

The $L$-shell processes required are

$$
2 l^{q} 3 l^{\prime p} n^{\prime \prime} l^{\prime \prime}+h \nu \rightarrow 2 l^{q-1} 3 l^{\prime p} n^{\prime \prime} l^{\prime \prime}+\mathrm{e}^{-}
$$

and

$$
2 l^{q} 3 l^{\prime p} n^{\prime \prime} l^{\prime \prime}+h \nu \rightarrow 2 l^{q-1} 3 l^{\prime p} n^{\prime \prime} l^{\prime \prime} n^{\prime \prime \prime} l^{\prime \prime \prime} \rightarrow 2 l^{q-1} 3 l^{\prime p} n^{i v} l^{i v}+\mathrm{e}^{-},
$$

where $3 l^{\prime p}$ stands for for $3 \mathrm{~s}^{s} 3 \mathrm{p}^{t} 3 \mathrm{~d}^{u}$ with $p=s+t+u$. Calculations are made for $q=1$ to 8 for $p=0$ and $q=8$ for $p>0$; e.g., up to $p=2$ for iron.

The $M$-shell processes required are simpler:

$$
3 l^{q} n^{\prime} l^{\prime}+h \nu \rightarrow 3 l^{q-1} n^{\prime} l^{\prime}+\mathrm{e}^{-}
$$

and

$$
3 l^{q} n^{\prime} l^{\prime}+h \nu \rightarrow 3 l^{q-1} n^{\prime} l^{\prime} n^{\prime \prime} l^{\prime \prime} \rightarrow 3 l^{q-1} n^{\prime \prime \prime} l^{\prime \prime \prime}+\mathrm{e}^{-},
$$

and are included only for iron, with $q=1$ to 3 .

To attain as much accuracy as possible for consistency with the existing $R$-matrix data, our perturbative calculations retain configuration interaction within the $\mathrm{N}$ electron (core) complex and the $(N+1)$-electron complex (for $\left.n^{\prime}=n^{\prime \prime}\right)$ : for example, we retain interactions between states such as $2 \mathrm{~s}^{2}{ }^{1} \mathrm{~S}$ and $2 \mathrm{p}^{2}{ }^{1} \mathrm{~S}$. For most cases, calculations are made both in $L S$-coupling and in intermediate coupling (including Breit-Pauli terms). Even when not significant for inner-shell contributions to opacity, this resolution is required for modelling non-LTE photoionized plasmas.

In table 2, we summarize the inner-shell calculations that we have carried-out. We have examined the convergence with respect to the inclusion of inner-shell data and estimate that the inclusion of further data would change the Rosseland means by less than $1 \%$.

The new data resulted-in an additional 2575458 level-resolved photoexcitation lines and 187351 total photoionization cross sections, i.e. summed-over all final states, contributing to the OP opacities. Much more data are archived, viz. final-state resolved photoionization cross sections and data for transitions and lines than were already included in the original OP work. 


\subsection{Database issues}

Given that we have already generated, and will be generating, large amounts of atomic data, some thought has been given to how it might be effectively archived for applications other than the one at hand. In particular, the Atomic Data and Analysis Structure (ADAS) [25] has long had the capability of handling radiation fields [26] but has, until recently, concentrated on utilizing escape factors [27]. The collisional-radiative modelling of finite-density non-LTE photoionized plasmas with ADAS requires that we archive final-state resolved photoionization data, summedover final channel angular momenta. We have written codes (ADASPE and ADASPI) to process the large amount of energy levels, radiative rates, autoionization rates and photoionization cross sections produced by AUTOSTRUCTURE and have defined such a suitable archive data structure of final-state resolved photoexcitation-autoionization and direct photoionization data, specifically, adf38 and adf39 ADAS data formats [25]. This partial data is then further reduced to total photoionization data for use by opacity calculations. For completeness, outer-shell photoionization data is archived in the adf39 files as well as inner-shell, but it is not used in the work reported-on here.

\section{Free-free transitions}

Contributions from free-free transitions are, in most cases, calculated in a hydrogenic approximation using the code of [28] which employs fits to accurate results from [29]. Allowance for Debye screening can be of importance at high density and is included if it gives increases in Rosseland-means by more than $0.1 \%$. The screening contribution is calculated using the Born-Elwert theory $([19,30,31])$. It is checked that, without screening, the Born-Elwert approximate gives agreement with the accurate results from [28] and [29] to better than $1 \%$ for all cases considered in the present work. For $\log (R) \leq-1$ and $\log (T) \leq 7.7$ it is found that inclusion of screening for free-free transitions never changes the Rosseland-mean by more than 2 or $3 \%$.

The correction for Debye screening was not included in the work of SYMP but is included in the present work.

\section{Results for Rosseland-mean opacities}

\subsection{Use of the variable $\log (R)$}

It is convenient to use the variable

$$
R=\rho / T_{6}^{3},
$$

where $\rho$ is the mass density in $\mathrm{g} \mathrm{cm}^{-3}$ and $T_{6}=10^{-6} \times T$, with $T$ in $\mathrm{K}$. For a solar mix, comparisons of $\log \left(\kappa_{\mathrm{R}}\right)$ from OP and OPAL for $\log (R)=-1$ to -6 were given in Figure 15 of SYMP, which showed the OPAL opacities to be larger than those from OP for larger values of $\log (R)$ with $\log (T)>5.5$.

\subsection{The 6-element mix}

Iglesias and Rogers in IR95 considered the importance of inner-shell transitions for a mixture of 6 elements $(\mathrm{H}, \mathrm{He}, \mathrm{C}, \mathrm{O}, \mathrm{S}$ and $\mathrm{Fe}$ ) with abundances chosen to be such as to give opacities similar to those for the complete solar mix. The adopted numberfractions, $f_{k}$, are given in Table 3 . 
Paper IR95 gave results for one temperature-density point, $\log (T)=6$ and $\log (R)=-2$ giving $\log (\rho)=-2$. OPAL calculations were made both with and without the inclusion of inner-shell processes. The inclusion of those processes was found to give an increase in $\kappa_{\mathrm{R}}$ by $30 \%$.

\subsection{OP results both with and without inner-shell transitions}

Figure 3 gives OP values of $\log \left(\kappa_{\mathrm{R}}\right)$ for the 6 -element mix, both with the inclusion of the inner-shell data discussed in Section 3 and without. The results without innershell data are essentially the same as those given in SYMP, but include some fairly minor improvements and use of the Q-EOS. Figure 4 shows $\delta \log \left(\kappa_{\mathrm{R}}\right)$, the increase in $\log \left(\kappa_{\mathrm{R}}\right)$ due to inclusion of the inner-shell data. For $\log (T)=6, \log (R)=-2$ we obtain an increase in $\kappa_{\mathrm{R}}$ by $31 \%$, in close agreement with the increase obtained in IR95.

Changes in $\kappa_{\mathrm{R}}$ due to changes in the EOS are much smaller than those due to the inclusion of inner-shell data: thus for $\log (T)=6, \log (R)=-2$, use of the Q-EOS in place of the EOS used in SYMP reduces $\kappa_{\mathrm{R}}$ by only $1.6 \%$ (in both cases, without inner-shell data).

\subsection{Results from $\mathrm{OP}$ and $\mathrm{OPAL}$}

Figure 5 shows values of $\log \left(\kappa_{\mathrm{R}}\right)$ for the 6-element mix from OPAL (data obtained from the OPAL website [32]) and from the present OP work with the inclusion of inner-shell data. It is seen that, for the larger values of $\log (R)$ where the inner-shell data is important, OP is now in close agreement with OPAL.

There are some remaining differences between OP and OPAL at smaller values of $\log (T)$ and $\log (R)$ where inner-shell data are not important. A feature at $\log (T) \simeq 5.2$, often referred to as the ' $Z$-bump', was first identified by Iglesias, Rogers and Wilson [33] as due to inclusion of very large numbers of $M$-shell transitions for various ionization stages of iron. For $\log (R) \leq-3$ there are seen to be some differences between OPAL and OP in the vicinity of that feature. Figure 6 shows, on a much more expanded scale, percentage difference for $\kappa_{\mathrm{R}},(\mathrm{OP}-\mathrm{OPAL})$, for $\log (R)=-3$ and -6 . For $\log (R)=-3$ the $Z$-bump feature from OP is seen to be broader than that from OPAL, and to have a peak value lower by about $10 \%$. For $\log (R)=-6$ the OP peak value is lower by $20 \%$. We plan to consider these differences further in a later paper.

\section{Discussion}

IR95 was mainly concerned with illustrative results for the case of the 6-element mix and $\log (T)=6, \log (\rho)=-2$. Plots of monochromatic opacities were given for C, S, Fe and for the mixture. Similar plots were obtained in the course of the present work.

\subsection{Density dependence}

It is seen from Figure 4 that the inner-shell contributions are most important at high densities. That can be understood by considering the case of high temperatures (similar considerations apply for other regions). At high temperatures, say $\log (T) \geq$ 7.0, the most important transitions are for iron $K$-shell. At the very lowest densities 
considered, the iron is almost fully ionized and there are no $K$-shell contributions. Then, as the density increases, one has $K$-shell transitions of the type

$$
1 \mathrm{~s}+h \nu \rightarrow \kappa \mathrm{p},
$$

and

$$
1 \mathrm{~s}^{2}+h \nu \rightarrow 1 \mathrm{~s} \kappa \mathrm{p},
$$

where $\kappa=n$ for transitions to a bound state and $\kappa=k$ for photoionization. Such transitions are included in the original OP calculations. With a further increase in density, one has states of the type $1 \mathrm{~s}^{2} C$ (where ' $C$ ' stands for states of outer electrons) and $K$-shell transitions of the type

$$
1 \mathrm{~s}^{2} C+h \nu \rightarrow 1 \mathrm{~s} C \kappa \mathrm{p} .
$$

Such transitions were not included in the original OP work but are included in the present work (see section 3).

\subsection{Line profiles}

Pressure broadening of all spectrum lines should be included, for both transitions to true bound states and for those to autoionizing states.

The importance of pressure-broadening for the iron $K$-shell is illustrated in Figure 7, which gives plots of $\sigma(u)$ for iron for $\log (T)=7$ and $\log \left(N_{\mathrm{e}}\right)=22.5$ and 25: for the 6-element mix, those values of $N_{\mathrm{e}}$ correspond to $\log (R)=-4.21$ and -1.71 . It is seen that, for the low-density case, there are very many resolved spectrum lines but that at higher densities pressure broadening leads to the lines being almost completely blended. We use the pressure-broadening theory from [35] and [18] and line-blending theory from [36].

We note that, beyond the $K$-edge, the cross sections are nearly the same for both densities, since both depend mainly on $K$-shell ionization. At the lower energies, say $u \leq 7$, the background opacity is mainly due to free-free transitions, giving crosssections per atom proportional to the electron density.

\subsection{Autoionization}

The Autostructure calculations give line widths due to radiation damping and to autoionization. We find, in practice, that inclusion of those contributions to the line profiles is never very important since widths due to pressure broadening are generally much larger: omission of autoionization widths would never give errors in Rosselandmeans larger than one on two percent.

\subsection{Fine-structure}

In both the OPAL and OP work, it was found that inclusion of fine-structure for outer-shell transitions could be of importance for the calculation of Rosseland-means (see [34] and SYMP). Most of our AUTOSTRUCTURE calculations were made both in $L S$-coupling (no fine-structure) and in intermediate coupling (with fine-structure). Test runs showed that inclusion of fine-structure for the inner-shell transitions never increased Rosseland-means by as much as $1 \%$, which was, again, a consequence of the importance of pressure-broadening. All final results as reported in the present paper were made with omission of inner-shell fine-structure. 


\section{The solar centre region}

Convection occurs throughout much of the solar interior and in those regions a precise knowledge of the Rosseland-mean opacity is not of great importance. However, convection does not occur in the deepest layers of the solar interior. Knowledge of the Rosseland-mean for the centre region is of importance for the construction of solar models, which can be tested against data from helioseismology.

At the solar centre, models give $\log (T)=7.196, \log (\rho)=2.179$ and $\log (R)=$ -1.409 (see [37, 38]). Opacities calculated using a number of different codes [39] show quite a large scatter. The most accurate values currently available are undoubtedly those from OPAL [40]. For the 6-element mix, opacities for the centre region from OP are a little larger than those from OPAL: by $2.3 \%$ at $\log (T)=7.2$ and $\log (R)=-1.5$.

The present work permits some further discussion of the solar-centre problem. In the OP work we use a mesh of values of $\log (T)$ and $\log \left(N_{\mathrm{e}}\right)$. We select a meshpoint $\log (T)=7.2, \log \left(N_{\mathrm{e}}\right)=26$ giving, for the 6-element mix, $\log (\rho)=2.294$ and $\log (R)=-1.306$. Figure 8 shows $\log (\sigma)$ for the mixture at that point. There are three main contributions to the centre opacity.

\subsection{Free-free}

The free-free contribution is mainly due to electron collisions with $\mathrm{H}^{+}$and $\mathrm{He}^{+2}$. The free-free cross section behaves like $u^{-2}$ and the process therefore dominates at low frequencies. We include Debye screening (see Section 4) which modifies the solarcentre opacities by about 1 or 2 per cent. OPAL include some further refinements (see [13]) but they are not likely to be of much importance.

\subsection{Electron scattering}

At lower densities, the electron-scattering cross section is equal to the Thomson cross section, and is independent of frequency. For higher densities, the cross section is modified by plasma collective effects. Both OP and OPAL use the theory of Boercker [41], and also allow for relativistic corrections.

\subsection{Atomic transitions}

In Figure 8 there is a just-discernible feature at $u \simeq 2$ due to sulphur $K$-shell transitions, and a much more conspicuous one at $u \simeq 5$ due to iron $K$-shell transitions. For hydrogenic iron, the $\mathrm{Ly}_{\alpha}$ line is at $u=5.10$ and the Lyman continuum starts at

$u=6.80$. Table 3 gives ionization fractions and ground-state occupation probabilities for iron: all stages up to $i=9$ are seen to contribute to the $K$-feature. It is seen from Figure 7 that the high $K$-shell lines are completely blended. It may be noted that the mean opacity will not be sensitive to the exact distribution amongst ionization stages (the $\phi_{i}$ of Table 4) since the cross sections for promotion of $1 \mathrm{~s}^{2}$ electrons will be much the same for the different stages.

\subsection{Use of a different frequency variable}

Equation (4) may be replaced by

$$
\frac{1}{\sigma_{\mathrm{R}}}=\int_{v=0}^{v_{\max }} \frac{1}{\sigma(u)} \mathrm{d} v
$$


where

$$
v(u)=\int_{u=0}^{v} F(u) \mathrm{d} u
$$

and $v_{\max }=v(u \rightarrow \infty)$ - numerical integrations give $v_{\max }=1.0553$. Figure 9 shows $1 / \sigma$ plotted against $v$ for the case of Figure 7 . The advantage of using Figure 9 is that it shows the sensitivity of $1 / \sigma_{\mathrm{R}}$ to the various features in $\sigma(u)$. There are seen to be rather small contributions from regions of small $u$ where $\sigma(u)$ is large but much more important contributions from large $u$ where $\sigma(u)$ is small.

\section{Summary}

Rosseland-mean opacities $\kappa_{\mathrm{R}}$ from the Opacity Project, OP, were originally found to be smaller than those from the OPAL project at high temperatures and high densities. Iglesias and Rogers, in IR95 [13], discussed the case of $\log (T)=6, \log (\rho)=-2$ where $\kappa_{\mathrm{R}}(\mathrm{OPAL})$ was larger than $\kappa_{\mathrm{R}}(\mathrm{OP})$ by about $30 \%$. They made two criticisms of the OP work: (a) OP calculated occupation probabilities $W$ using a Holtsmark MFD where, at high densities, it is a poor approximation; (b) OP omitted some important inner-shell atomic data.

\subsection{Occupation probabilities}

In the present work we calculated values of $W$ using expressions from Nayfonov et al [16], who used an MFD theory that is valid to high densities. It is shown that the expressions for $W$ given in [15] and [16] are not valid in the limit of very high densities: a simple expedient removes that difficulty. It is found that final results for Rosselandmean opacities are not very sensitive to the adopted occupation probabilities.

It is noted that the values of $W$ obtained from the OPAL work seem to be rather surprisingly large for more highly-excited states.

\subsection{Inner-shell data}

New opacity calculations have been made for the 6-element mix introduced in IR95. Extensive inner-shell atomic data were computed using the code AUTOSTRUCTURE for the 6-element mix introduced in IR95. It is shown that inclusion of those data removes all major differencies between the OP and OPAL work.

\subsection{Future work}

In the work described in SYMP, opacities were calculated for 17 cosmically-abundant chemical elements. Work is now in progress to obtain inner-shell data for those elements not included in the present work. 


\section{References}

[1] Schwarzschild M 1958 Structure and evolution of the stars, Princeton

[2] Cox J P and Giuli R T 1968 Principles of stellar structure, 2 vols., Gordon and Breach

[3] Collins G W 1989 Fundamentals of stellar structure, Freeman

[4] Simon N R 1982 Astrophys. J. 260 L87

[5] Iglesias C A, Rogers F J and Wilson B G 1987 Astrophys. J. 322 L45

[6] Iglesias C A and Rogers F J 1996 Astrophys. J. 464943

[7] Seaton M J 1987 J. Phys. B: At. Mol. Phys. 206363

[8] Seaton M J, Yu Yan, Mihalas D and Pradhan A K 1994 Mon. Not. R. astr. Soc. 266805 (SYMP)

[9] The Opacity Project Team, The Opacity Project, IOP Publishing, Vol I, 1995: Vol. II, 1997

[10] Rogers F J, Wilson B G and Iglesias C A 1988 Phys. Rev. A 385007

[11] Kurucz R L 1990 Trans. IAU XXb 1168

[12] Lynas-Gray A E, Storey P J and Seaton M J 1995 J. Phys. B: At. Mol. Opt. Phys. 282817

[13] Iglesias C A and Rogers F J 1995 Astrophys. J. 443469 (IR95)

[14] Badnell N R 1987 J. Phys. B: At. Mol. Phys. 203827

[15] Hummer D G and Mihalas D 1988 Astrophys. J. 331794

[16] Nayfonov A, Däppen W, Hummer D G and Mihalas D 1999 Astrophys. J. 526451

[17] Hooper C F 1966 Phys. Rev. 14977 and 1968 Phys. Rev. 165215

[18] Seaton M J 1990 J. Phys. B: At. Mol. Opt. Phys. 233255

[19] Bethe H A and Salpeter E E 1997 Quantum mechanics of one-and two-electron atoms Plenum

[20] Ballance C P, Badnell N R and Berrington K A 2001 J. Phys. B: At. Mol. Opt. Phys. 343287

[21] Pindzola M S, Badnell N R and Griffin D C 1992 Phys. Rev. A 465725

[22] Condon E U and Shortley G H 1935 Theory of Atomic Spectra, CUP

[23] Eissner W, Jones M and Nussbaumer H 1974 Comput. Phys. Commun. 8270

[24] Badnell N R 1997 J. Phys. B: At. Mol. Opt. Phys. 301

[25] Summers H P 2003 ADAS User Manual Version 2.6 webpage http://adas.phys.strath.ac.uk

[26] Burgess A and Summers H P 1969 Astrophys. J. 1571007

[27] Fischbacher G A, Loch S D and Summers H P 2002 Astron. Astrophys. 389295

[28] Hummer D G 1988 Astrophys. J. 327484

[29] Karzas W J and Latter R 1961 Astrophys. J. Suppl. Ser. 6167

[30] Elwert G 1939 Ann. d. Physik 34178

[31] Rozsnyai B F 1979 J. Quant. Spect. Rad. Trans. 22337

[32] webpage: www-phys.llnl.gov/Research/OPAL/index.html

[33] Iglesias C A, Rogers F J and Wilson B G 1987 Astrophys. J. 322 L45

[34] Iglesias C A, Rogers F J and Wilson B G 1992 Astrophys. J. 397717

[35] Seaton M J 1988 J. Phys. B: At. Mol. Phys. 213033

[36] Däppen W, Anderson L and Mihalas D 1987 Astrophys. J. 319195

[37] Guenther D B, Demarque P, Kim Y-C and Pinsoneault M H 1992 Astrophys. J. 387372

[38] Bahcall J N and Ulrich R K 1988 Rev. Mod. Phys. 60297

[39] Rose S J 2001 J. Quant. Spect. Rad. Trans. 71635

[40] Iglesias C A and Rogers F J 1991 Astrophys. J. 371408

[41] Boercker D B 1987 Astrophys. J. 316 L95 
Table 1. Occupation probabilities $\left(W_{n}\right)$ and average volumes $\left(V_{n}\right)$ for $\mathrm{C}^{5+}$ at

$T=10^{6} \mathrm{~K}$ and $N=9.7 \times 10^{21} \mathrm{~cm}^{-3}$.

\begin{tabular}{llll}
\hline$n$ & $N V_{n}$ & $W_{n}(\mathrm{OPAL})$ & $W_{n}(\mathrm{Q})$ \\
\hline 1 & $2.08(-4)^{a}$ & 1.000 & 1.000 \\
2 & $6.67(-3)$ & 0.996 & 0.997 \\
3 & $6.31(-2)$ & 0.995 & 0.967 \\
4 & 0.330 & 0.995 & 0.705 \\
5 & 1.216 & 0.914 & 0.154 \\
6 & 3.562 & 0.527 & $1.58(-2)$ \\
7 & 8.875 & 0.162 & $1.87(-3)$ \\
8 & $1.96(+1)$ & $2.37(-2)$ & $2.82(-4)$ \\
9 & $3.96(+1)$ & $2.23(-3)$ & $5.22(-5)$ \\
\hline
\end{tabular}

a $2.08(-4)=2.08 \times 10^{-4}$.

Table 2. Inner-shell transitions considered: $Z$ is the nuclear charge of the element and $N$ the number of electrons left on the final ion.

\begin{tabular}{|c|c|c|c|c|c|c|c|c|}
\hline$Z$ & $N$ & \multicolumn{2}{|c|}{ Shells } & $Z$ & $N$ & \multicolumn{3}{|c|}{ Shells } \\
\hline 2 & 1 & $K$ & & & & & & \\
\hline \multirow[t]{4}{*}{6} & 1 & $K$ & & 26 & 1 & $K$ & & \\
\hline & 2 & $K$ & & & 2 & $K$ & & \\
\hline & 3 & $K$ & $L$ & & 3 & $K$ & $L$ & \\
\hline & & & & & 4 & $K$ & $L$ & \\
\hline \multirow[t]{4}{*}{8} & 1 & $K$ & & & 5 & $K$ & $L$ & \\
\hline & 2 & $K$ & & & 6 & $K$ & $L$ & \\
\hline & 3 & $K$ & $L$ & & 7 & $K$ & $L$ & \\
\hline & & & & & 8 & $K$ & $L$ & \\
\hline \multirow[t]{10}{*}{16} & 1 & $K$ & & & 9 & $K$ & $L$ & \\
\hline & 2 & $K$ & & & 10 & & $L$ & \\
\hline & 3 & $K$ & $L$ & & 11 & & $L$ & $M$ \\
\hline & 4 & $K$ & $L$ & & 12 & & $L$ & $M$ \\
\hline & 5 & & $L$ & & 13 & & & $M$ \\
\hline & 6 & & $L$ & & & & & \\
\hline & 7 & & $L$ & & & & & \\
\hline & 8 & & $L$ & & & & & \\
\hline & 9 & & $L$ & & & & & \\
\hline & 10 & & $L$ & & & & & \\
\hline
\end{tabular}


Table 3. Number fractions $\left(f_{k}\right)$

for the 6-element $(k)$ mix of

Iglesias and Rogers [13].

\begin{tabular}{ll}
\hline$k$ & $f_{k}$ \\
\hline $\mathrm{H}$ & $9.071(-1)$ \\
$\mathrm{He}$ & $9.137(-2)$ \\
$\mathrm{C}$ & $4.859(-4)$ \\
$\mathrm{O}$ & $9.503(-4)$ \\
$\mathrm{S}$ & $9.526(-5)$ \\
$\mathrm{Fe}$ & $3.632(-5)$ \\
\hline
\end{tabular}

Table 4. Ionization fractions $\left(\phi_{i}\right)$ and ground-state occupation probabilities $\left(W_{0}\right)$ for iron for $\log (T)=7.2$ and $\log \left(N_{\mathrm{e}}\right)=26$.

\begin{tabular}{lll}
\hline$i$ & $\phi_{i}$ & $W_{0}$ \\
\hline 0 & 0.000 & 1.000 \\
1 & 0.001 & 1.000 \\
2 & 0.039 & 1.000 \\
3 & 0.155 & 0.959 \\
4 & 0.289 & 0.954 \\
5 & 0.282 & 0.941 \\
6 & 0.162 & 0.930 \\
7 & 0.058 & 0.918 \\
8 & 0.012 & 0.894 \\
9 & 0.002 & 0.872 \\
10 & 0.000 & 0.847 \\
\hline
\end{tabular}




\section{Figure captions}

(a)

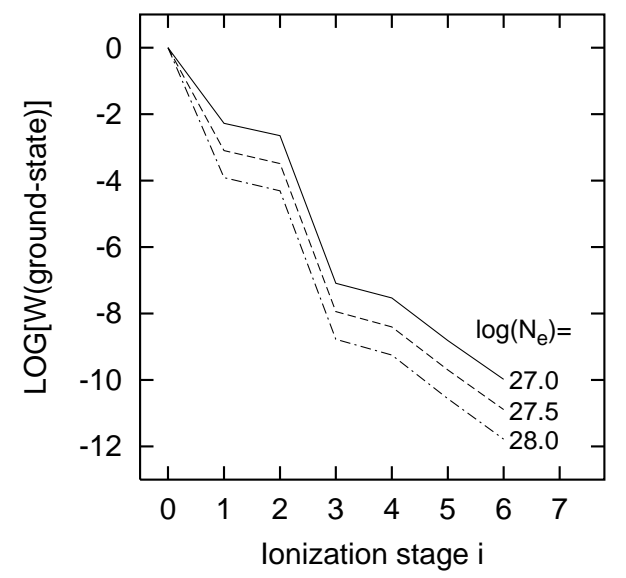

(b)

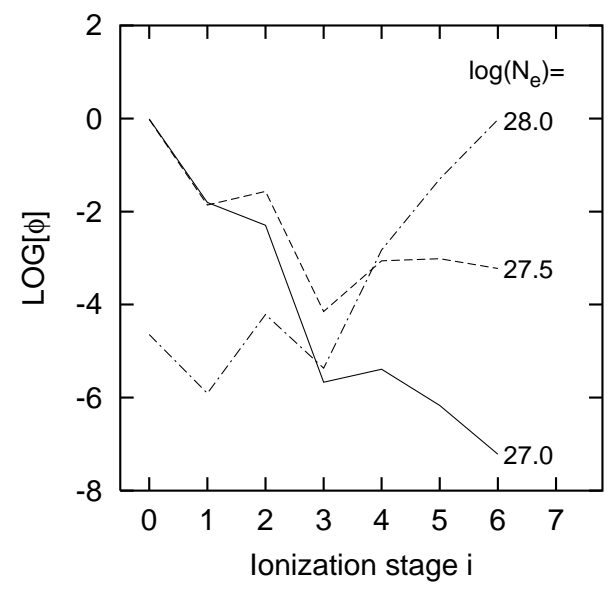

Figure 1. Carbon at $\log (T)=7.5$ and $\log \left(N_{\mathrm{e}}\right)=27.0,27.5$ and 28.0. (a) Groundstate occupation probabilities, $W$, against ionization stage $i$. (b) Ionization fractions, $\phi_{i}$, calculated without a cut-off, $W_{\mathrm{c}}$, in $W$. 


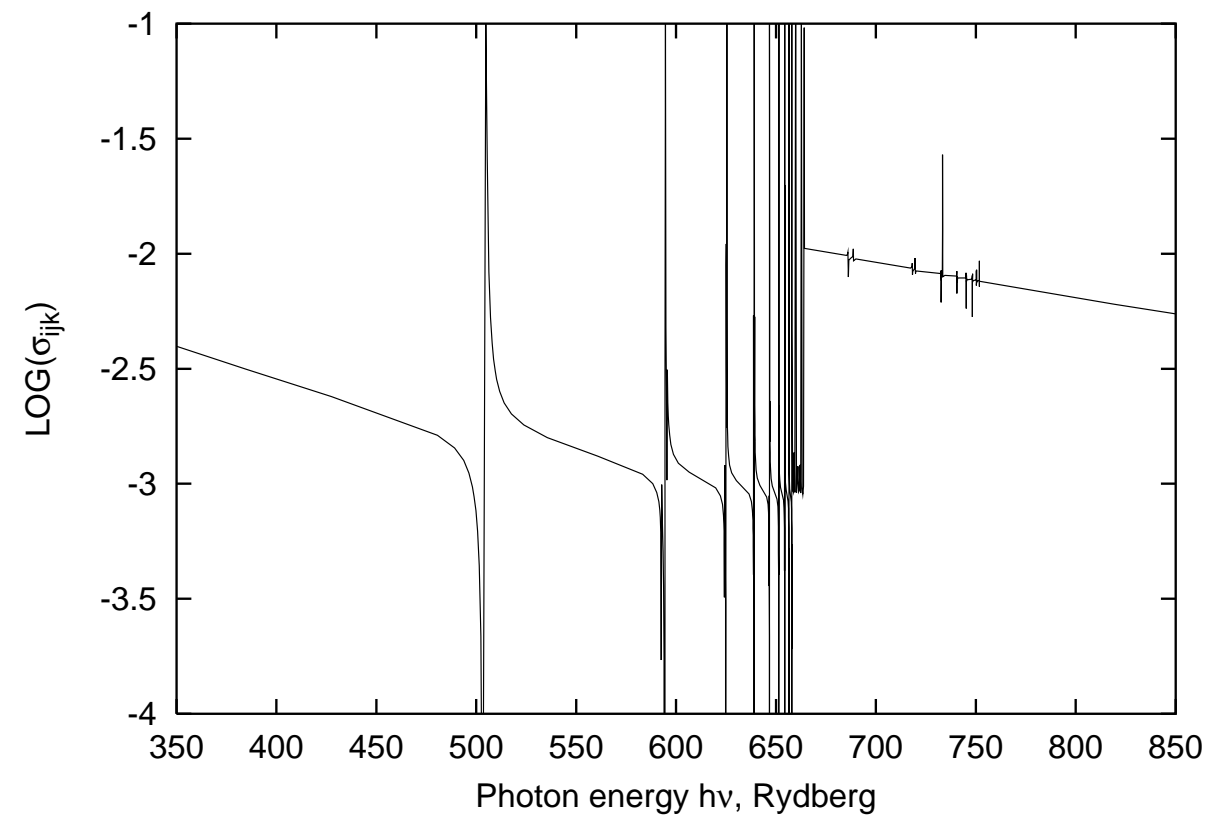

Figure 2. The atomic cross-section, $\sigma_{i j k}$, for photoionization from $\mathrm{Fe}^{24+} 1 \mathrm{~s} 2 \mathrm{~s}{ }^{1} \mathrm{~S}$, in the vicinity of the $K$-edge. Cross section in atomic units. 


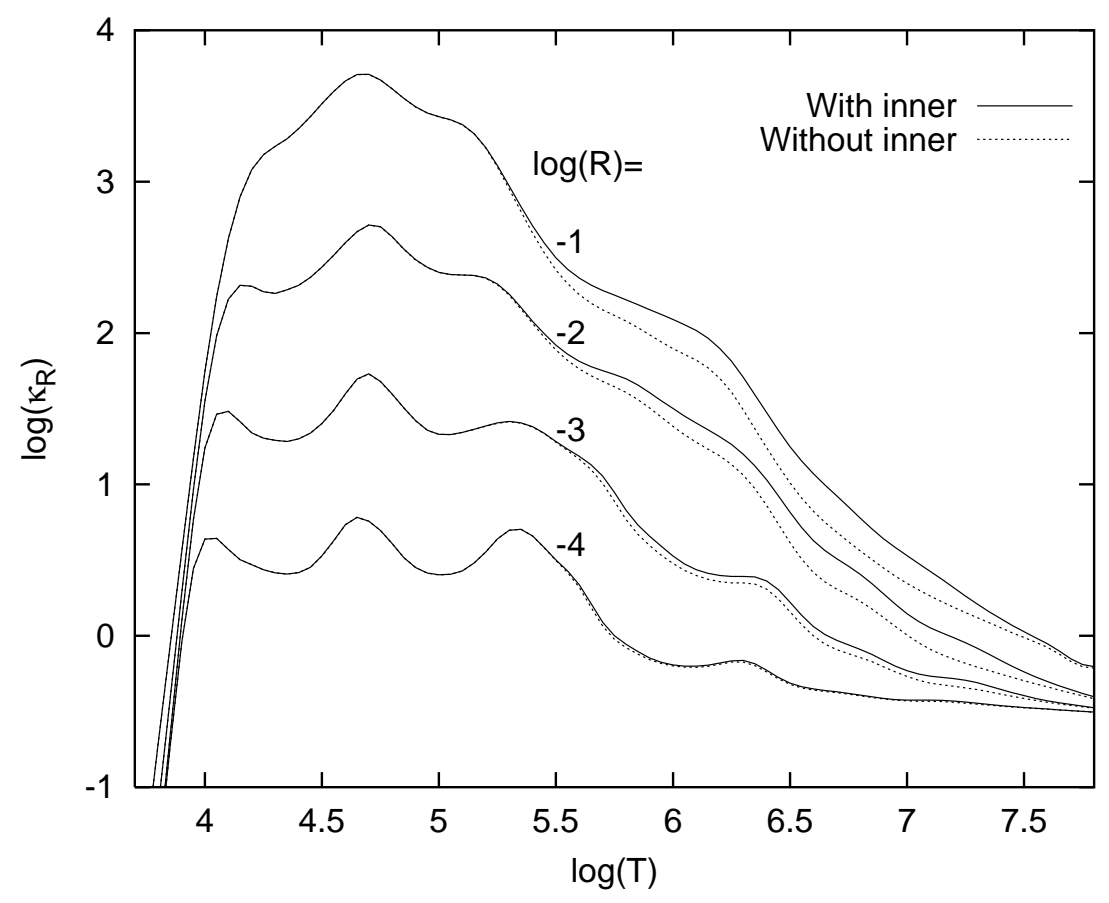

Figure 3. OP Rosseland-mean opacities for the 6-element mix for $\log (R)=-1$, $-2,-3$ and -4 , with inclusion of inner-shell data (full lines) and without those data (dotted lines). The opacities are in cgs units: $\mathrm{cm}^{2} \mathrm{~g}^{-1}$. 


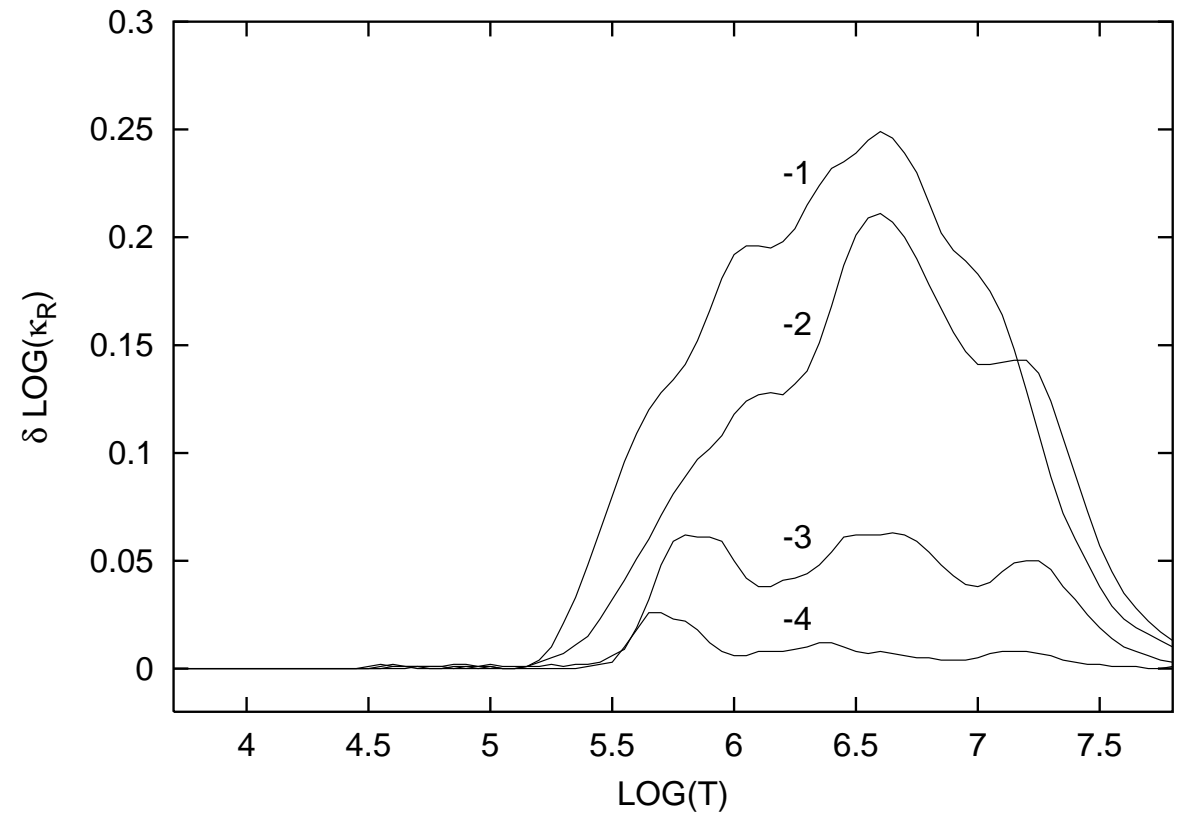

Figure 4. $\delta \log \left(\kappa_{\mathrm{R}}\right)$, the change in Rosseland-mean opacity which results from the inclusion of inner-shell data. 


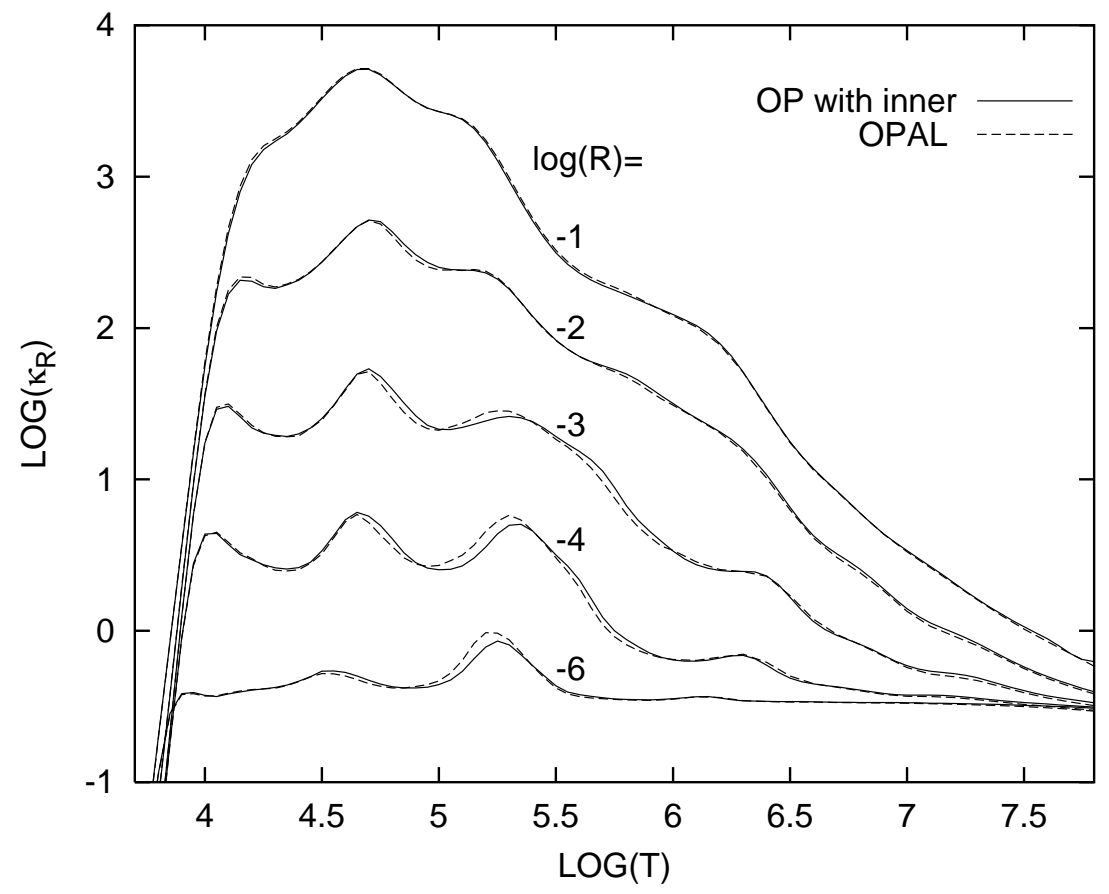

Figure 5. Rosseland-mean opacities for the 6-element mix, with the inclusion of inner-shell data: full lines, OP, present work; dashed lines, OPAL, from [32]. Opacities in cgs units. 
(a)

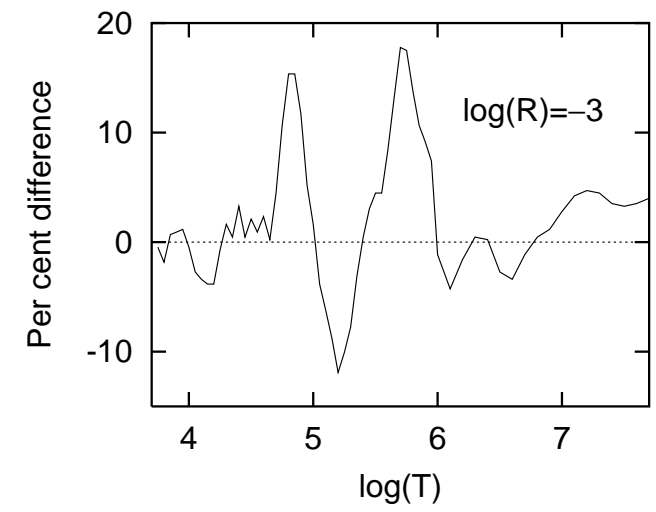

(b)

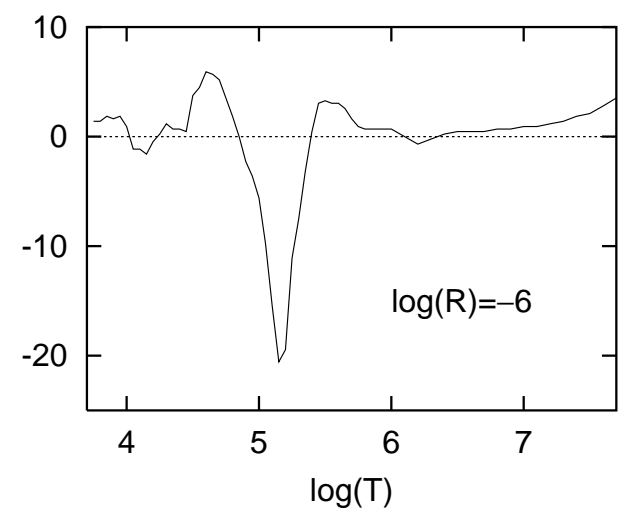

Figure 6. Percentage differences between OP and OPAL Rosseland means, OP-OPAL. (a), $\log (R)=-3$. (b), $\log (R)=-6$. 
(a)

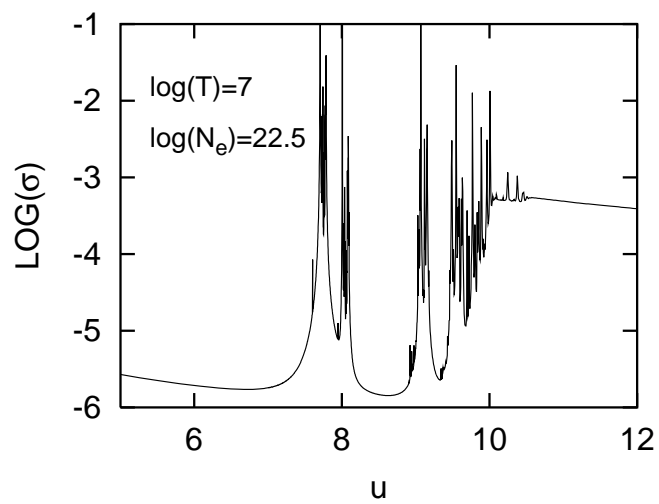

(b)

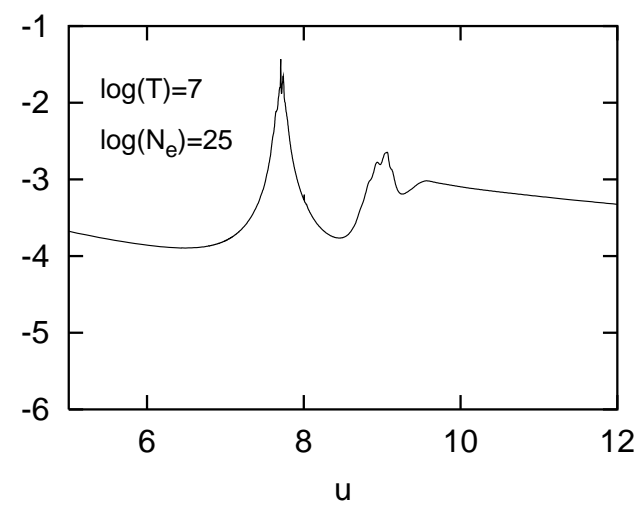

Figure 7. The opacity cross-section, $\sigma$, for iron in the vicinity of the $K$-edge, at $\log (T)=7$ : cross section in atomic units. Pressure broadening included.

(a) $\log \left(N_{\mathrm{e}}\right)=22.5$, and (b) $\log \left(N_{\mathrm{e}}\right)=25$. 


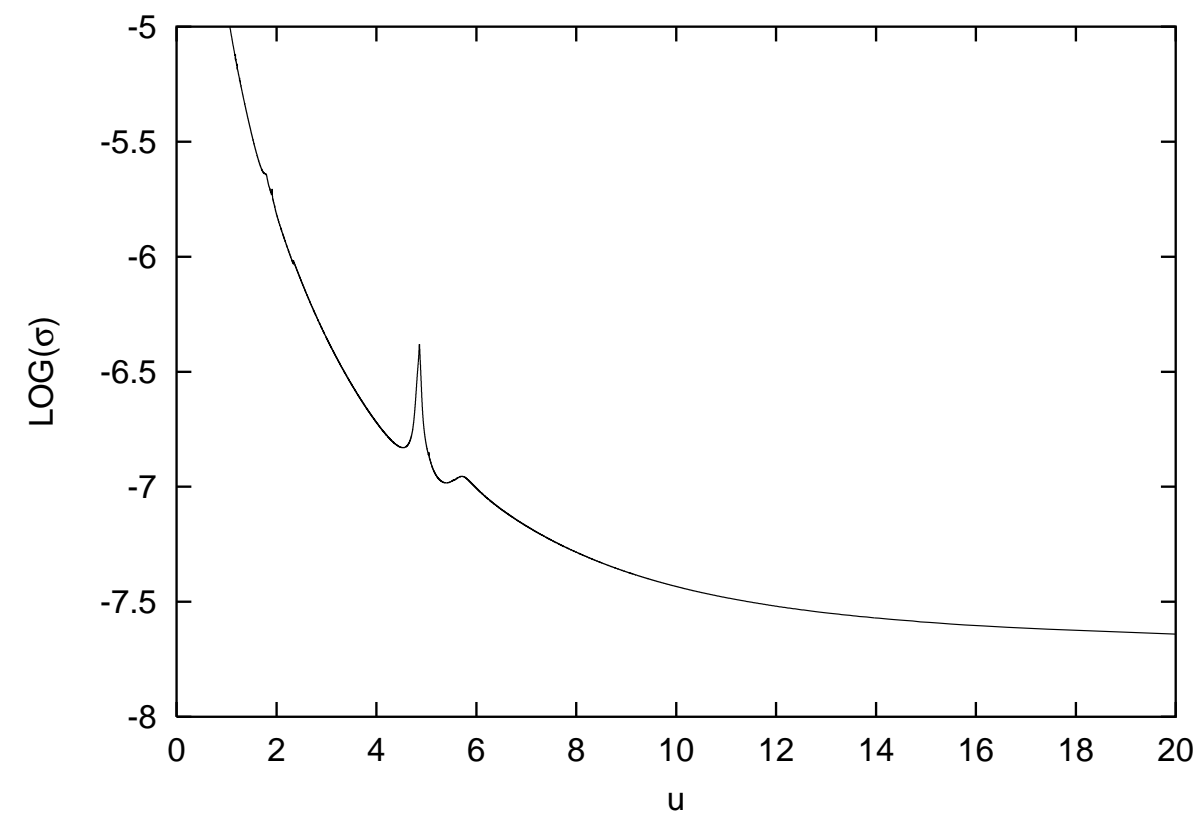

Figure 8. The opacity cross-section, $\sigma$, (in atomic units) for the 6-element mix at $\log (T)=7.2, \log \left(N_{\mathrm{e}}\right)=26, \log (R)=-1.306$. 


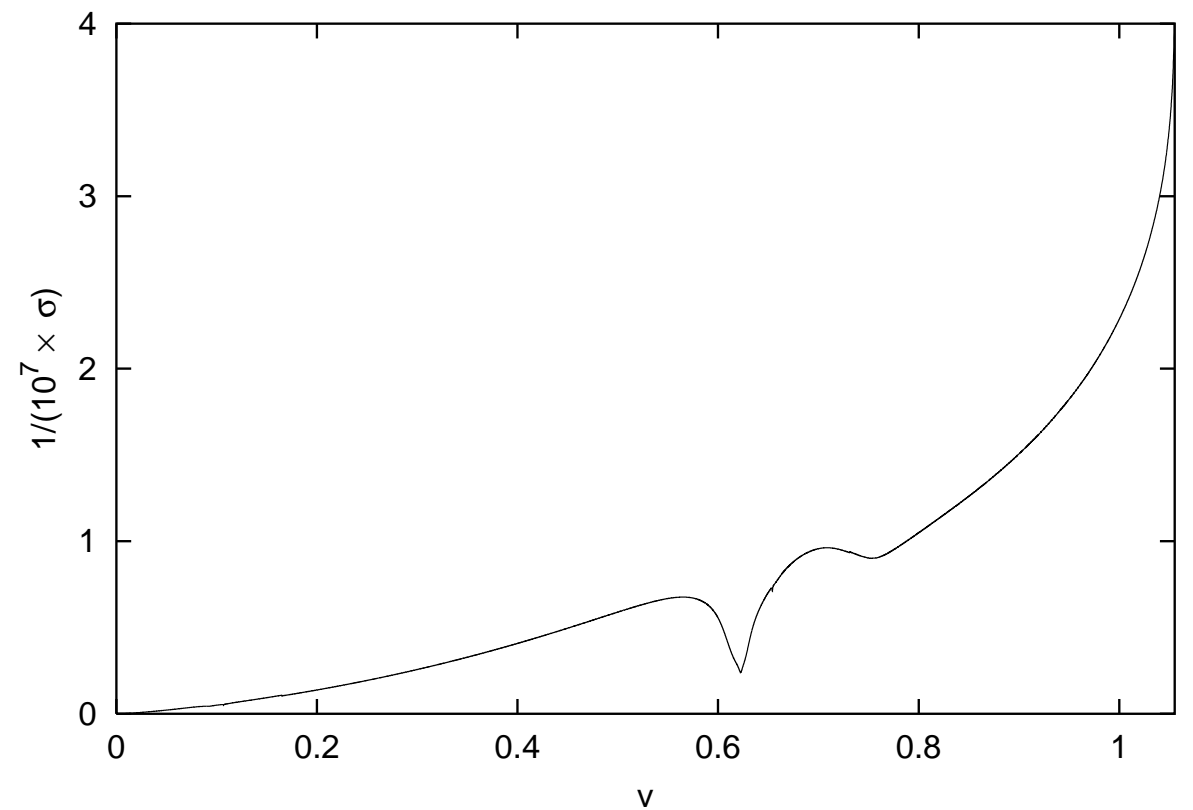

Figure 9. $1 / \sigma$ against $v$, defined by equation (35), for the case of Figure 7 . 